\title{
Effect of grain disorientation on early fatigue crack propagation in FCC polycrystals: dislocation dynamics simulations and corresponding experimental validation
}

\author{
C. Robertson ${ }^{\mathrm{c}}$, G.V.PrasadReddy ${ }^{\mathrm{a}, \mathrm{b}}$, C. Déprés ${ }^{\mathrm{a}}$, M.Fivel $^{\mathrm{d}}$ \\ aaboratorySYMME, Université de Savoie, BP80439,74944 Annecy-le-Vieux Cedex, France \\ ${ }^{b}$ Indira Gandhi Centre for Atomic Research, Kalpakkam, Tamil Nadu 603102 INDIA \\ ${ }^{\mathrm{c}} \mathrm{CEA}, \mathrm{DEN}$, Service de Recherche Métallurgiques Appliquées, F91191 Gif-sur-Yvette, \\ France \\ ${ }^{\mathrm{d}}$ SIMaP-GPM2, Grenoble INP, CNRS/UJF, 101 Rue de la Physique, BP 46, 38402 St Martin \\ d'Hères cedex, France
}

\begin{abstract}
3-dimensional dislocation dynamics (DD) simulations are performed, in face-centred cubic bi-crystals, to study the microcrack interaction with first microstructural barrier under high cycle fatigue (HCF) loading conditions. Based on experimental observations, we presumed that microcracks are blocked by grain boundaries and that subsequent propagation/transmission occurs by the growth of surface relief in a secondary grain adjoining the primary crack. This mechanism is herein called indirect transmission and is found to strongly depend on grain-to-grain disorientation. A semi-analytical model proposed earlier is discussed with the DD simulation results in the context of first-barrier compliance. The proposed model describes the documented experimental results related to the effect ofgrain size, grain misorientation and microcrack propagation kineticsin fatigued 316L steel polycrystals.
\end{abstract}

\subsection{Introduction}

In face-centered cubic (FCC) single-phase polycrystals, with no defects and/or precipitates, the initiation of stage-I fatigue cracks usually occurs in the surface grains. Prior to the microcrack initiation, significant surface markings develop in the form of extrusions concomitant with the formation of persistent slip bands (PSBs) that form the basis for the nucleation of microcracks [1-3]. In subsequent to the microcrack initiation, microcrack propagatesuntil it encounters the first microstructural barrier (i.e. a grain boundary). As a result, the subsequent crack propagation or retardation not only depends strongly on the loading amplitude, but also on the next (or secondary) grain orientation [4] defined by the tilt, twist and theta angles (as per refs. [5,6]).

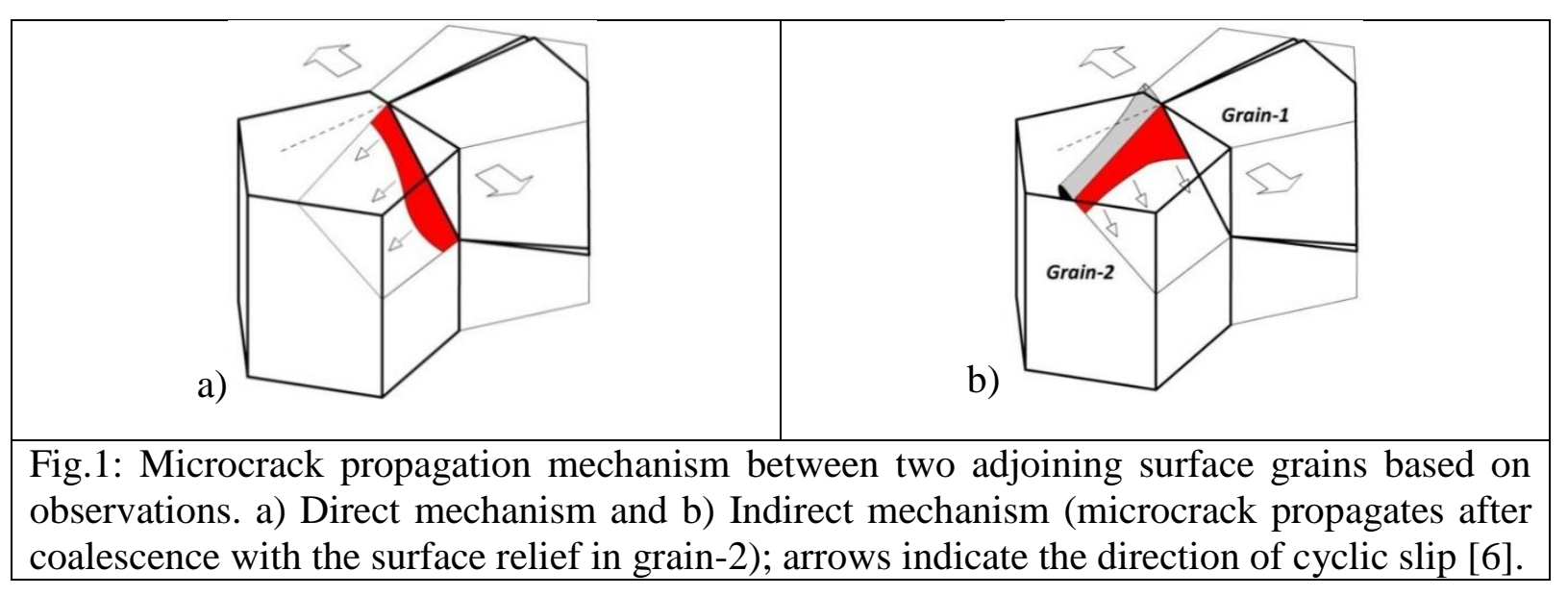


This paper briefly presents a micro-mechanical model and its application in terms of microcrack growth prediction, and also its validation with the experimental results, in the context of the experimentally observed indirect transmission mechanism. Therefore, our simulations do not apply to prior or subsequent crack development stages and therefore substantially differ from all the previouscrack-tip plasticity investigations [7-9].

\subsection{Dislocation dynamics simulation setup 2.1 Simulation geometry and parameters}

The DD-code used for this study is an edge-screw model called TRIDIS, developed at SIMaP laboratory [11]. The material parameters used for austenitic stainless steelare given elsewhere [6]. The implementation of thermally-activated cross-slip is done as per the explanation in $[12,13]$.A pseudo bi-crystal aggregate simulation setup (Fig.1b) is considered to investigate the indirect crack transmission mechanism. In Fig.1b, grain-1 is denoted as primary grain and it includesan arrested crackand no dislocations. It is important to mention that grain-1 geometry can be chosen arbitrarily and has no effect on this study. The pentagonal shape shown in Fig. 1 is adopted for simplicity. The DD simulations are carried out in secondary grain(i.e. grain-2) and both grains are defined as 5-faceted cylinders. The grainaxis is aligned perpendicularly to the gain free surface(see Fig.1). Dislocations gliding in grain-2 can escape through the top surface of simulated volume, whereas all the other surfaces act as strong andimpenetrable obstacles. Each dislocation segment leaving the crystal through the top boundary produces a step in the corresponding surface [14].Image forces are not implemented in our simulations, since their influence on PSBslip activity isnoticed to be negligible[15],even in the presence of a crack [16].The initial, grain-2 dislocation microstructure consists of 24 Frank-Read sources,2-sources on each of the 12 FCC slip systems $a / 2<110>\{111\}$. The sources are simply placed (inside grain-2) within $1 \mu \mathrm{m}$ of the arrested crack tip and evolve according to the local, effective stress conditions (including the primary crack stress field). The DD simulations are carried out with a fully reversed cyclic strain for applied plastic strain ranges of $\Delta \varepsilon_{\mathrm{p}}=10^{-4}$ and $2 \times 10^{-4}$ compatible with $\mathrm{HCF}$ conditions.The applied stress level is monitored and controlled as detailed in [14]. All the simulations are performed under isothermal conditions at $300 \mathrm{~K}$.

\subsection{Implementation of heterogeneous crack stress field}

The microcrack in the grain-1 induces a long-range stress field in the surrounding elastic medium comprising grain-2. The effect of crack stress field is treated using the superimposition principle where the total stress applied to each dislocation segment (within grain-2) is taken as the sum of: (i) applied stress (homogeneous stress), (ii) the dislocationinduced stress (internal stress), and (iii) the crack stress field (heterogeneous stress), calculated using Eqs.1-4 from reference [6]. DD simulations were performed for varying orientations of the grain-2, with respect to the crack plane. The simulation results are analysed by means of a semi-quantitative model, to be presented in the next section.

\subsection{Discussion:DD simulation based microcrack transmission model}

In response to the applied stress, the mobile dislocations introduced (in grain-2) near the crack front zone glide into several different parallel planes and gradually develop pile-ups at the grain boundary. It is possible to calculate the stress-strain response associated with the 
dislocation structures in crack tip region, by considering $\mathcal{N}$ slip planes ${ }^{1}$ for which the simplified analytical solution is [6]:

$$
\Delta \tau_{\text {local }, w c}=\frac{1}{\mathcal{N}}\left[\frac{1}{S} \frac{\mu}{(1-v)}\right] \Delta \gamma_{\text {local }, w c}
$$

where $\Delta \gamma_{\text {local,wc and }} \Delta \tau_{\text {local,wc }}$ are the shear strain and shear stress ranges actingin the crack tip region (the subscript implies: "local, with crack"), $\mu$ the shear modulus, the Poisson ratio and $\mathrm{S}$ is adimensionless parametercharacterizing the grain geometry[15,17].To derive the 'microcrack transmission semi-analytical model', the different terms in Eq. 1 will be evaluated separately, as explained in next two paragraphs.

Evaluation of $\Delta \tau_{\text {local,wc: }}$ The shear stress rangein the crack tip region is given by:

$$
\Delta \tau_{\text {local }, w c} \approx \frac{\Delta K_{0}}{\sqrt{2 \pi r}}=\Delta \tau_{\text {global }, w c}^{*} \sqrt{\frac{\pi a}{2 \pi r}}
$$

where $\Delta \tau_{\text {global,wc }}^{*}$ is shear stress accumulated beyond the yield point, at the individual grain scale (and thus the subscript"global"). By equating the crack size $a \sim D_{g}$, on the presumption of fully cracked primary grain-1, one obtains:

$$
\Delta \tau_{\text {local }, w c} \approx \Delta \tau_{\text {global }, w c}^{*} \sqrt{\frac{D_{g}}{2 r}}
$$

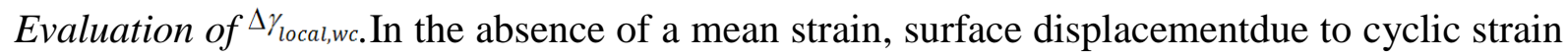
accumulationis given by [14]:

$$
\gamma^{\text {surf }} \propto \eta \Delta \gamma_{p} \sqrt{N}
$$

where $\Delta \gamma_{\mathrm{p}}$ is the imposed plastic shear-strain range, $N$ the cycle number and $\eta$ is a dimensionless factor depending on simulation-setup and material parameters (see ref. [14] for the derivation of Eq.4 usingDD simulations). On the other hand, in the presence of a mean strain (compression $\neq$ tension), the accumulation of cyclic surface displacement is given by:

$$
\gamma^{\text {surf }} \propto \eta \Delta \varepsilon_{p}\left(1+2 \frac{\overline{\gamma_{p}}}{\Delta \gamma_{p}}\right) \sqrt{N}
$$

The Eq.5is derived based on a series of cyclic simulations, where the level of mean strain $\overline{\gamma_{p}}$ is systematically changed, and the corresponding results plus physical interpretation were given in reference [18].However, in the present study, to evaluate the quantity $\overline{\gamma_{p}}$ due to the primary crack,two separate DD simulations using a fixed plastic strain range $\Delta \gamma_{p}$ were performed. Firstly, a simulation is carried out without the influence of crack stress field (i.e. no crack) and generated a reference $\gamma_{\text {woc }}^{\text {surf }}(N)$ curve. This curve is thenfitted with Eq.4, which is then subsequently solved to obtain a fixed $\eta$ value. The second simulation isperformed in the presence of acrack in grain-1 and the results are used to generate a $\gamma_{w c}^{\text {surf }}(N)$ curve. This new curve is thenfitted using Eq.5, andit is assumedthat the differences between $\gamma_{w c}^{\text {surf }}$ and $\gamma_{w o c}^{\text {surf }}$ are due to the mean strain $\overline{\gamma_{p}}$ acting in the crack tip region. Theobtained $\overline{\gamma_{p}}$ valueisthen finally inserted in Eq.1, assuming that $\Delta \gamma_{\text {local }, w c}=\bar{\gamma}_{p}$.

\footnotetext{
${ }^{1}$ The number of active slip planes $\mathcal{N}$ must not be confused with the number of cycles $N$.
} 
Application of semi-analytical modelto indirect microcrack transmission.

InsertingEq.3 in the left-hand term of Eq.1 andrearranging the different termsgives:

$$
\frac{\mathrm{D}_{\mathrm{g}}}{(2 \mathrm{r})}=\left(\frac{1}{\mathcal{N}}\left[\frac{1}{S} \frac{\mu}{(1-v)}\right] \frac{\Delta \gamma_{\text {local }, w c}}{\Delta \tau_{\text {global }, w c}^{*}}\right)^{2}
$$

Eq.6is applied to various DD simulations conductedusingvaryingtwist anglesfor a fixed cyclic loading level $\Delta \gamma_{\mathrm{p}}=10^{-4}$. Each grain disorientation case gives: i) a given $\Delta \gamma_{\text {local,wc }} / \Delta \tau_{\text {global,wcratio,to }}^{*}$ be called the first-barrier compliance and ii)a specific curve in Fig.2a. In general, microcrack transmission is a local process and takes place at the scale of individual shear bands in polycrystals [14]. Therefore, the surface displacements developed by individual shear bands need to be calculated in order to evaluate the microcrack transmission kinetics based on the indirect mechanism.From the above mentioned Eq.4 and Eq.5, the evolution of surface displacement in the wholecrack tip zoneis characterized by:

$$
\frac{\gamma_{w c}^{\text {surf }}}{\gamma_{\text {woc }}^{\text {surf }}}=\left(1+2 \frac{\overline{\gamma_{p}}}{\Delta \gamma_{p}}\right)
$$

The surface displacement generated bya single shear band can be obtained bythe ratio of'right-hand term of Eq. 7'to'the total number of active shear-bands $\mathcal{N}$ '. The latter can be obtained by solvingEq.6, yielding:

$$
\frac{\gamma_{w c}^{\text {surf }}}{\gamma_{\text {woc }}^{\text {surf }}}=\frac{\sqrt{\frac{\mathrm{Dg}}{2 \mathrm{r}}}}{\left[\frac{1}{s(1-v)}\right]\left(\frac{\Delta \gamma_{\text {local }, w c}}{\Delta \tau_{\text {global } w c}^{*}}\right)}\left(1+\sqrt{\frac{\mathrm{D}_{\mathrm{g}}}{2 \mathrm{r}}}\right)
$$

Eq. 8 thus describes the accumulation of average surface displacementby single shear bandsin grain-2, ahead of the primary crack front.

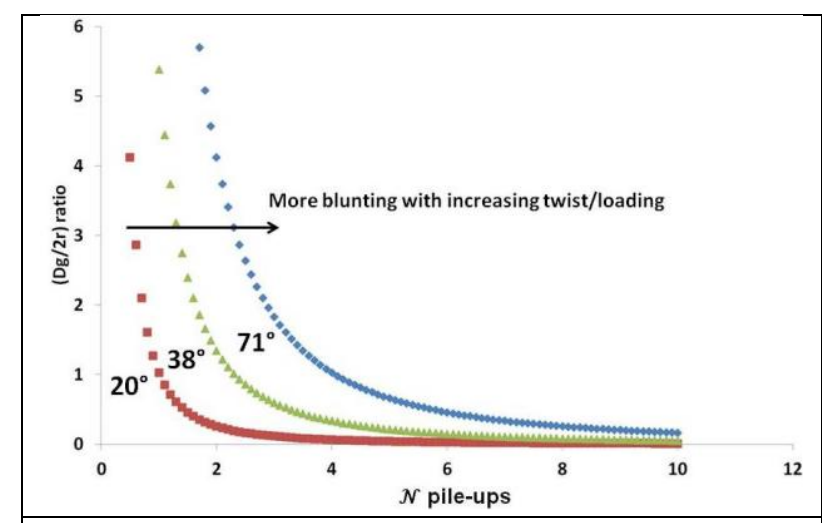

(a)

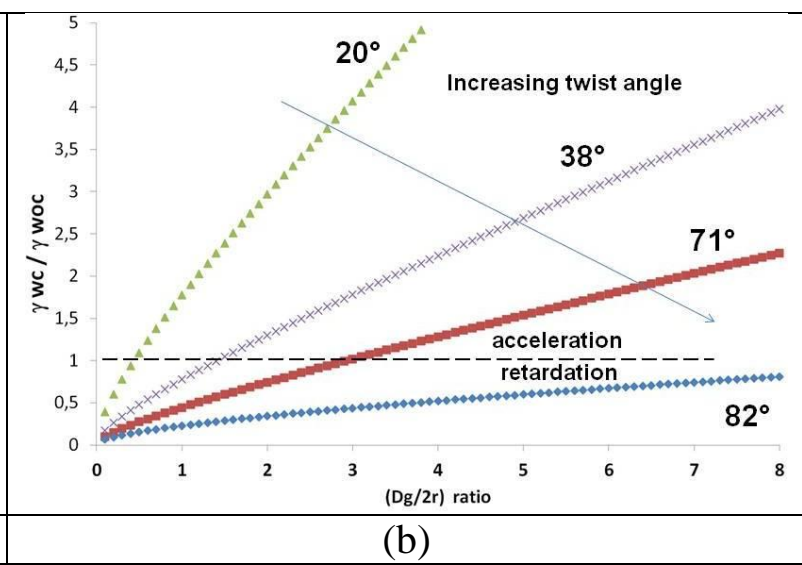

(b)

Fig.2: Model results. (a) Variation of parameter $\left(\mathrm{D}_{\mathrm{g}} / 2 \mathrm{r}\right.$ i.e. reciprocal half-distance to the crack tip) as a function of 'number of active pile-ups $\mathcal{N}$ in the crack process zone'; increase in twist angle increases the plastic zone size.(b) Microcrack transmission kinetics towards the secondary grain as a function of twist angle; the results are normalized w.r.to the non-cracked case. A slip ratio of $\gamma_{w c}^{\text {surf }} / \gamma_{\text {woc }}^{\text {surf }}>1$ means extrusion growth acceleration and $<1$ implies extrusion growth retardation in the presence of crack. Twist angles $=20^{\circ}, 38^{\circ}, 71^{\circ}$ and $82^{\circ}$ are used for the same tilt-angle at $24^{\circ} \pm 2^{\circ}[6]$.

Eq.8expression helps in analysing the simulation results and also in making direct comparisons between the simulation and experiment. For instance, itspredictions are compared with microcrack kinetics observed in actual polycrystals, where the crystallographic 
orientations of primary and secondary grains(and corresponding twist angle) are evaluated using electron back-scattering diffraction technique.

The crack initiation time $\mathrm{N}_{\mathrm{A} 2}$ and the crack arrest time $\mathrm{T}_{1>2}$ are evaluated by observation of replicas extracted at different number of cycles. A correlation/equivalence can be drawn between the Fig.2 and Fig.3. Fig.2 indicates that plasticity spreading onto multiple slip bands increase with the twist angle, leading to less net surface relief. This in turn implies an increase in crack arrest time at primary grain boundary and also a simultaneous increase in crack initiation time, in the secondary grain. This can be seen from Fig.3 wherein large twist angles corresponding to low $1 / \sqrt{\mathrm{T}_{1>2} / \mathrm{N}_{\mathrm{A} 2}}$ values denote a delay in crack transmission kinetics and conversely, at small twist angles.

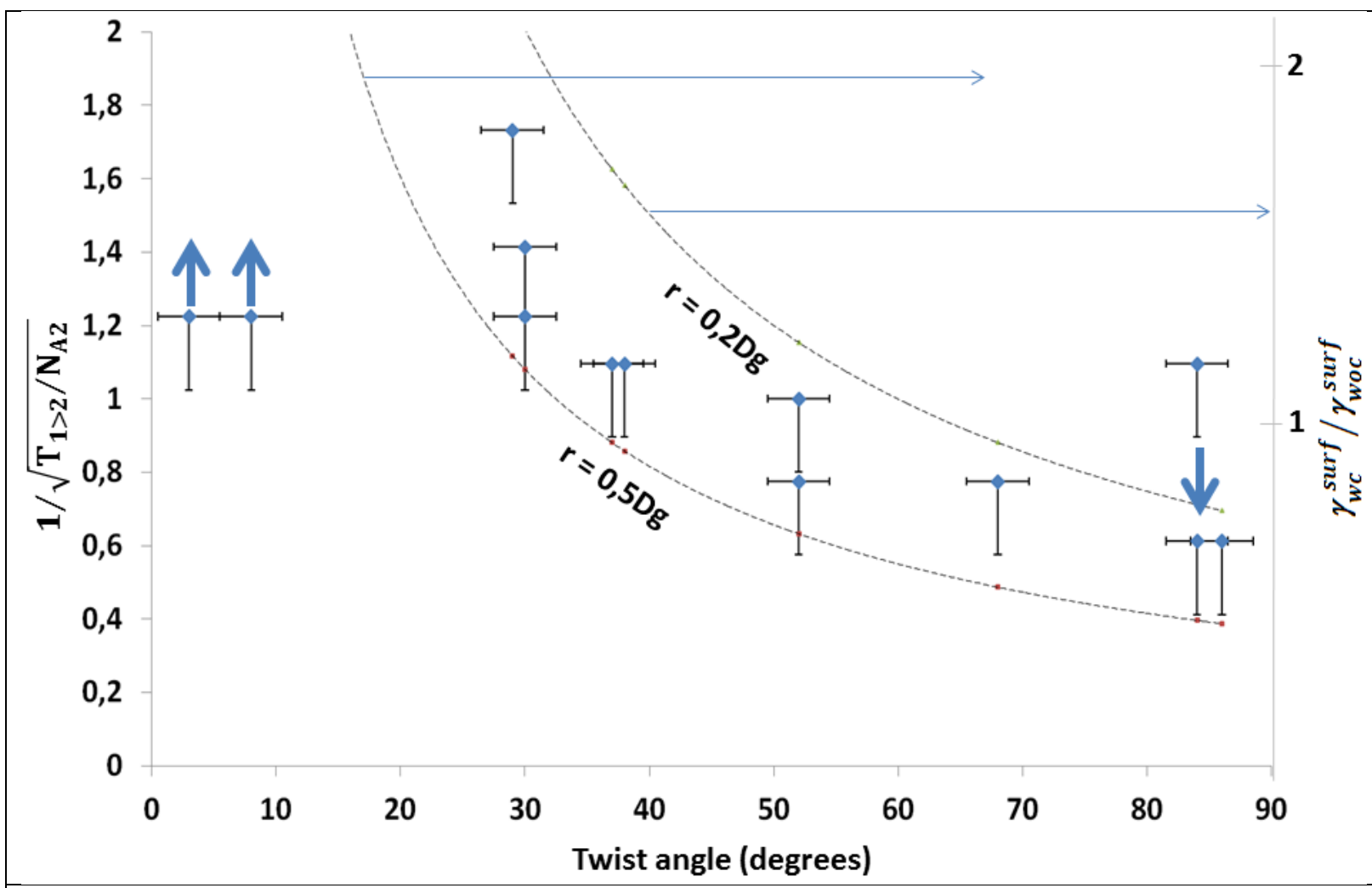

Fig.3:Microcrack propagation kinetics from primary to secondary grain: effect of twist angle. The micro-mechanic model predictions (dashed curves plottedfor different distances $r$ with respect to the primary crack tip region: $r=0.2 \mathrm{D}_{\mathrm{g}}$ and $\mathrm{r}=0.5 \mathrm{D}_{\mathrm{g}}$ where $\mathrm{Dg}$ is the grain diameter) are directly compared with experimental observations.More specifically, $\gamma_{w c}^{\text {surf }} / \gamma_{w o c}^{\text {surf }}$ ratio calculated using Eq.8is directly comparedto the $1 / \sqrt{\mathrm{T}_{1>2} / \mathrm{N}_{\mathrm{A} 2}}$ quantity. Theabove two quantities are equivalent based on Eq.4 and Eq.5 and [14]; assuming thatmicrocrack initiation takes place whenever $\gamma_{w c}^{\text {surf }}$ or $\gamma_{\text {woc }}^{\text {surf }}$ achieve a critical value, depending on the material, temperature and environmental conditions.

\subsection{Conclusions/Summary}

3-dimensional DD simulations performed in FCC bi-crystals to study the microcrack interaction with first microstructural barrier, under high cycle fatigue (HCF) loading conditions, provided interesting results. At first, DD simulations revealed that a cyclic slip localization or dispersion onto several slip bands is found to strongly depend on the twist angle. Our semi-analytical model developed based on the cyclic plasticity ahead of the crack 
front is able to capture the above cyclic slip distribution. The model also predicts that an increase in twist angle (for a given $\mathrm{D}_{\mathrm{g}} / 2 \mathrm{r}$ ratio) increases the development of active slip planes $\mathcal{N}$ in the crack process zone. The DD simulation results fairly show equivalence with the results of crack initiation/arrest times obtained from actual experiments.

\subsection{Acknowledgements}

The authors acknowledge the financial support of the French National Agency for Research Agency through theAFGRAP project.

\subsection{References}

[1] Basinski Z S, and Basinski S, J Prog Mater Sci 36 (1992) 89.

[2] Man J, Obrtlik K, Blochwitz C and Polak J, Acta Mater 50 (2002) 3767.

[3] Man J, Obrtlik K and Polak J, Phil Mag A 89 (2009) 1295.

[4] Christ H J, Duber O, Fritzen C P, Knobbe H, Köster P, Krupp U, and Künkler B, Comput Mater Sci 46 (2009) 561.

[5] Zhai T, Wilkinson A J and Martin J W, Acta Mater 48 (2000) 4917.

[6] Prasad Reddy G V, Robertson C, Déprés C and Fivel M, Acta Mater 61 (2013) 5300.

[7] Hansson P. Int J Fatigue 31 (2009) 1346.

[8] Deshpande V S, Needleman A and Van der Giessen E, Acta Mater 51 (2003) 4637.

[8] Van der Giessen E, Deshpande V S, Cleveringa H H M, and Needleman A J, Mechanics and Physics of Solids, 49 (2001) 2133.

[10] Espinosa H D, Panico M, Berbenni S and Schwarz K W, Int J Plasticity 22 (2006) 2091.

[11] Verdier M, Fivel M and Groma I, Mod Sim Mater SciEng 6 (1998) 755.

[12] Déprés C, Robertson C, and Fivel M, Phil Mag A 84 (2004) 2257.

[13] Robertson C, Fivel M, and Fissolo A, Mat SciEng A 315 (2001) 47.

[14] Déprés C, Robertson C, and Fivel M, Phil Mag A 86 (2006) 79.

[15] Déprés C, Modélisation physique des stades précurseurs de l'endommagement en fatigue dans l'acier inoxydable 316L,DoctoralThesis, Institut National Polytechnique de Grenoble, France, 2004.

[16] Déprés C, Prasad Reddy G V, Robertson C, and Fivel M, Phil Mag A 94 (2014) 4115.

[17] Hirth J P, and Lothe J. Theory of Dislocations, McGraw-Hill (1982) p 770

[18] Robertson C, Déprés C, and Fivel M, Special Issue of Trans. Indian Institute of Metals, $63(2010) 529$. 